\title{
Luminal Metastatic Breast Cancer: Current Concepts and Future Approaches
}

\author{
Johannes Ettl \\ Department of Gynecology and Obstetrics, Klinikum Rechts der Isar, Technical University Munich (TUM), \\ Munich, Germany
}

Among all patients with metastatic breast cancer the luminal subtype accounts for more than two thirds of all cases. The mainstay of treatment is endocrine based systemic therapy. Numerous publications and therapy recommendations have concluded that chemotherapy is not the right option for endocrine sensitive disease, except in rare situations of visceral crises [1]. Recently published data both from randomized clinical trials and from realworld registries provide evidence that in the metastatic HR-positive/HER2-negative setting about half of our patients do not survive more than 4 years [2,3]. Even though the metastatic disease cannot be cured, disease stabilization for a long period of time can be achieved. This also implicates that for many of our patients not only the disease burden but also the load of a lifelong therapy is to be taken care of with a maximum of clinical expertise. Thus, quality-of-life issues play a key role. The issue of highquality research in order to bring the field forward is not only about developing new drugs against the disease; with the huge already existing armamentarium against luminal breast cancer, it is also about determining the best sequence of therapy and the best method of therapy surveillance. Notably these points will unlikely be clarified by traditional clinical research. New methodologies to generate clinical evidence in the field need to be recognized. Gathering evidence outside of clinical trials using patientreported data in the environment of social media and big data is one of the potential ways to bring us forward.
The focus of this issue is on metastatic luminal breast cancer. Current concepts which impact the treatment of patients with HR-positive HER2-negative metastatic disease are being reviewed by invited authors.

CDK4/6 inhibitors have become the standard of care in first-line treatment of metastatic luminal breast cancer. However, the majority of patients with metastatic luminal breast cancer will eventually develop resistance to this treatment. In their review article "Treatment of Luminal Metastatic Breast Cancer beyond CDK4/6-Inhibition: Is There a Standard of Care in Clinical Practice?" Mavratzas and Marmé [4] give an overview over potential subsequent therapies including future approaches which are being tested in current clinical trials.

A newly available treatment option for luminal metastatic breast cancer with mutations in the PIK3CA gene is alpelisib, an oral PI3K-inhibitor, which has shown efficacy in PIK3CA-mutated tumors in combination with fulvestrant. Following FDA approval in 2019, alpelisib became available in Germany through an early-access program. In their research article Hester et al. [5] report on first experiences with this new treatment option in the clinical routine and point out the importance of patient education and an interdisciplinary approach when treating patients with alpelisib.

Real-world data on treatment outcomes and PRO in metastatic breast cancer are becoming increasingly important, since data from clinical trials are not available for 
all treatment situations. Systematically established realworld data can be used to support evidence particularly for rare treatment situations and subgroups. In their review article Lüftner et al. [6] describe the prospective academic translational research network PRAEGNANT as one of the existing data registries for metastatic breast cancer patients in a real-life setting.

Genetic and genomic testing has become therapeutically relevant in the treatment of metastatic breast cancer. It is also integral component within the luminal subtype. The role of personalized medicine has become increasingly prominent. Grill and Klein [6] review the current developments in genetic and genomic testing in the field of luminal metastatic breast cancer. They shed light on the new challenges and possibilities for the treatment of metastatic luminal breast cancer arising from personalized medicine.

I would like to thank all of the invited authors for their dedicated work and for sharing their expertise in the field of metastatic luminal breast cancer.

\section{Conflict of Interest Statement}

Personal fees/travel support was received from: Amgen, AstraZeneca, Celgene, DaiichiSankyo, Eisai, Lilly, Medivation, Novartis, Odonate, Pierre Fabre, Pfizer, Roche, Seagen, Tesaro, and Teva.

\section{References}

1 Cardoso F, Paluch-Shimon S, Senkus E, Curigliano G, Aapro MS, André F, et al. 5th ESOESMO international consensus guidelines for advanced breast cancer (ABC 5). Ann Oncol. 2020 Dec;31(12):1623-49.

2 Kobayashi K, Ito Y, Matsuura M, Fukada I, Horii R, Takahashi S, et al. Impact of immunohistological subtypes on the long-term prognosis of patients with metastatic breast cancer. Surg Today. 2016 Jul;46(7):821-6.
3 Sledge GW Jr, Toi M, Neven P, Sohn J, Inoue $\mathrm{K}$, Pivot X, et al. The effect of abemaciclib plus fulvestrant on overall survival in hormone receptor-positive, ERBB2-negative breast cancer that progressed on endocrine therapy: MONARCH 2 - A randomized clinical trial. JAMA Oncol. 2019 Sep 29;6(1):116-24.

4 Mavratzas A, Marmé F. Treatment of luminal metastatic breast cancer beyond CDK4/6- inhibition: Is there a standard of care in clinical practice? Breast Care. doi: 10.1159/000514561.

5 Hester A, Henze F, Travi C, Harbeck N, Würstlein R. First experiences with alpelisib in clinical routine: Case reports from a German breast center. Breast Care. doi: $10.1159 / 000514794$.
6 Lüftner D, Hartkopf AD, Lux MP, Overkamp F, Tesch H, Titzmann A, Pöschke P, Wallwiener M, Müller V, Beckmann MW, Belleville E, Janni W, Fehm TN, Kolberg HC, Ettl J, Wallwiener D, Schneeweiss A, Brucker SY, Fasching PA. Challenges and opportunities for real-world evidence in metastatic luminal breast cancer. Breast Cancer. DOI: 10.1159/000515701.

7 Grill S, Klein E. Incorporating genomic and genetic testing into the treatment of metastatic luminal breast cancer. Breast Care. doi: $10.1159 / 000513800$. 